\title{
Medicamentos de venta libre en el Brasil
}

\author{
Isabela Heineck, ${ }^{1}$ Eloir Paulo Schenkel ${ }^{1}$ y Xavier Vidal ${ }^{2}$
}

RESUMEN En el presente estudio se analizaron los 100 productos del mercado farmacéutico popular (de venta libre) más vendidos en el Brasil, en el período comprendido entre junio de 1992 y junio de 1993. Se excluyeron 23 productos alimentarios clasificados como medicamentos. La muestra, formada por 77 medicamentos, representaba aproximadamente $67 \%$ del consumo en valores y $76,8 \%$ del consumo en unidades de las especialidades vendidas en el mercado popular. Para la clasificación de los medicamentos, se utilizó el sistema anatómico-terapéutico-químico (ATC). Las especialidades también se clasificaron con la metodología del valor intrínseco, que considera la eficacia y los riesgos de las mismas. La mayoría de las especialidades (91\%) mostraron "poco valor intrínseco" (dudoso/nulo, relativo, inaceptable), y 70\% correspondieron a combinaciones en dosis fijas. Solo 10 especialidades pertenecían a la Relación Nacional de Medicamentos, producida por el Ministerio de Salud, y cuatro a la lista de medicamentos esenciales de la OMS. Los grupos terapéuticos a los que pertenecían las especialidades fueron, en orden de mayor a menor frecuencia, el aparato digestivo, la piel, el aparato genitourinario, el aparato musculosquelético, el sistema nervioso central, los antiparasitarios, el aparato respiratorio y los órganos de los sentidos. El subgrupo terapéutico de mayor venta fue el de preparaciones para la garganta, mientras que el que representó el mayor valor en ventas fue el de los analgésicos no opiáceos y antipiréticos. Los resultados confirman la hipótesis planteada de que los medicamentos de venta libre en el Brasil presentan mala calidad terapéutica y de que el consumo de muchas de estas especialidades farmacéuticas representa gastos innecesarios para la población. Se puede afirmar también que algunos de esos medicamentos no deberían estar disponibles para una población que no está al corriente de las complicaciones asociadas con su utilización, puesto que $25 \%$ de las intoxicaciones registradas en el país se deben a la automedicación. Una estrategia para mejorar la utilización de medicamentos a largo plazo es modificar la percepción que tiene la población en general con respecto a la necesidad y uso de los medicamentos para curar sus dolencias.

El Brasil es un país de contrastes: los privilegiados, $20 \%$ de la población, poseen ingresos 26 veces mayores que los de los habitantes más pobres, que también representan $20 \%$ del total (1). La calidad de los servicios de salud

\footnotetext{
1 Universidade Federal do Rio Grande do Sul, Programa de Posgrado, Facultad de Farmacia. Dirección postal: Av. Ipiranga, 2752, 90610-000 Porto Alegre RS, Brasil. Fax: (051)330-5610.

2 Universidad Autónoma de Barcelona, Departamento de Farmacología y Psiquiatría, Barcelona, España. Fax: (3)428-5112
}

que reciben estos grupos es marcadamente diferente. Solamente los privilegiados tienen recursos suficientes para ser atendidos en los servicios privados, que son comparables a los servicios de salud de los países desarrollados (2). Del resto de la población, los pocos que tienen acceso a los servicios de salud deben someterse al caos del servicio público, o, lo que es más común, "resuelven" sus problemas en el mostrador de las farmacias. Este difícil acceso a la atención médica lleva a la mayoría de las personas a automedicarse. Si lo hicieran solamente con medicamentos de venta libre y sobre la base de una orientación del farmacéutico, podría considerarse un proceso hasta cierto punto racional. Pero, según un estudio realizado en la ciudad de Belo Horizonte con una muestra de 106 farmacias, 98\% del personal que vende medicamentos no tiene capacitación técnica; solo en dos farmacias se exigía receta médica para la venta de aque- 
llos medicamentos no clasificados como de venta libre (3).

Tampoco existe en la población el concepto de que el medicamento es una sustancia que entrãna riesgos y que, por tanto, debería utilizarse con la orientación de un profesional capacitado para ello. Schenkel et al. comprobaron la venta de medicamentos en bares y fruterías en la ciudad de Porto Alegre, lo que muestra que el medicamento se consideraba un artículo de primera necesidad que, además, se utilizaba como los demás productos en venta en esas tiendas (4). Un agravante es la deficiencia del control estatal de los medicamentos, tanto en la fase de registro como en la de control después de su comercialización.

En 1985 en el Brasil, las cifras de consumo de medicamentos considerados ineficaces e incluso peligrosos fueron más altas que en otros países de América Latina. De todos los hepatoprotectores vendidos en países latinoamericanos, 38\% corresponden al Brasil, junto con $41,7 \%$ de todos los estimulantes del apetito, $45,5 \%$ de todas las combinaciones de vitamina $C$ con minerales y $65 \%$ de todos los psicoestimulantes (5). Un ejemplo de la falta de vigilancia posterior a la comercialización de los fármacos es el caso del misoprostol, indicado para el tratamiento de la úlcera péptica y que en el Brasil se está utilizando como abortivo $(6,7)$. Todos estos datos permiten postular que los medicamentos en ese país son de mala calidad terapéutica y de que el consumo de algunos de ellos, especialmente los que pertenecen al mercado popular (de venta libre), representa un gasto innecesario para la población, ya que esos medicamentos no están sujetos a los controles de comercialización y publicidad que rigen para los demás.

El objetivo general de este estudio sobre la utilización de los medicamentos es analizar cualitativa y cuantitativamente los 100 productos que más se venden en farmacias del mercado popular del Brasil. Para ello se establecen como metas: 1) clasificarlos según su valor intrínseco; 2) comprobar su presencia en la Relación Nacional de Medicamentos (RENAME) del Ministerio de Salud y en la lista de medica- mentos esenciales de la OMS; 3 ) determinar el número de principios activos en cada uno de ellos; y 4) identificar los grupos terapéuticos que más se consumen y los que representan las mayores ventas.

\section{MATERIALES Y MÉTODOS}

Los datos sobre el consumo de medicamentos, aportados por una muestra de farmacias o de grandes distribuidores de medicamentos, se obtuvieron de Intercontinental Medical Services del Brasil (IMS/Brasil/ 93), con sede en São Paulo. Se analizaron 77 de las 100 especialidades farmacéuticas del mercado popular que más se vendieron en el período de junio de 1992 a junio de 1993. Las otras 23 especialidades se excluyeron, por tratarse de productos higiénico-dietéticos, como leches especiales, edulcorantes y pastas dentífricas.

La composición de cada medicamento por nombre genérico se obtuvo del Dicionário Brasileiro de Especialidades Farmacêuticas (8), del Catálogo Brasileiro de Produtos Farmacêuticos (9) y de la información proporcionada por la industria de fármacos. Solamente interesaron para el análisis los principios activos. Los medicamentos se clasificaron por grupos terapéuticos según la clasificación anatómico-terapéuticoquímica (ATC) y las Guidelines for ATC classification publicadas por el Centro Colaborador de la OMS para la Metodología de las Estadísticas Farmacéuticas en Oslo, Noruega (10). En los casos de combinaciones a dosis fijas se asignó un código a cada principio activo y otro a la especialidad.

El valor intrínseco de los medicamentos se determinó según la metodología propuesta por Laporte, Porta y Capellà (11), acudiendo a la literatura médica (12-34) para evaluar la relación entre riesgos y beneficios. Según dicha metodología, los medicamentos se clasifican en cuatro categorías de acuerdo con su eficacia e inocuidad. Tienen gran valor aquellos medicamentos cuyo uso tiene indicaciones bien definidas, cuyos efectos son inmediatos y obvios, y cuya eficacia se ha demostrado mediante ensayos clínicos controlados. Los medicamentos tienen un valor relativo cuando contienen un principio activo de valor más otros ingredientes de eficacia terapéutica dudosa cuya adición al preparado no está respaldado por ningún dato obtenido en condiciones clínicas bien controladas. Estos medicamentos se consideran irracionales desde el punto de vista farmacológico y terapéutico. Los medicamentos sin valor o de valor dudoso son aquellos cuya eficacia no ha sido demostrada de manera convincente en ensayos clínicos controlados, pero que no han producido efectos nocivos graves o frecuentes que se hayan documentado. Finalmente, los medicamentos son inaceptables cuando la relación entre riesgos y beneficios es claramente desfavorable en toda circunstancia (11).

Se verificó si los medicamentos de la muestra estaban incluidos en la segunda edición de la RENAME y en la Octava Lista de Medicamentos Esenciales de la OMS $(35,36)$.

\section{RESULTADOS}

En el período de julio de 1992 a julio de 1993 el consumo de medicamentos del mercado popular en el Brasil (132842 000 unidades) representó $10,5 \%$ de todas las unidades de medicamentos vendidas y $6,7 \%$ (US\$ 249889000 ) del monto total de ventas. La muestra de los 77 medicamentos representa aproximadamente $67 \%$ (US\$ 168572000 ) del valor monetario en ventas y 76,8\% (102 117000 unidades) de las unidades farmacéuticas vendidas en el mercado popular.

\section{Composición de los medicamentos}

Cincuenta y cuatro especialidades (70\%) constituían combinaciones a dosis fijas. La media de principios activos por especialidad fue de 2,8; los medicamentos combinados, a exclusión de los monofármacos, tuvieron una media de 3,5 principios activos por especialidad. Los subgrupos terapéuticos cuyas especialidades presen- 
taron el mayor número de principios activos (alrededor de seis) fueron las vitaminas (A11) ${ }^{3}$, los tónicos (A13), los antibióticos y fármacos para uso dermatológico (D06) y las preparaciones nasales (R01). Ciertos subgrupos terapéuticos, como los productos tópicos para dolores musculares y articulares (M02) y los antitusígenos y medicamentos antigripales (R05), se compusieron de combinaciones a dosis fijas exclusivamente.

Entre los analgésicos (N02) se observaron más combinaciones que fármacos simples. Se consumieron 9311000 unidades $(82 \%)$ de analgésicos combinados (cuatro especialidades) y solamente 2083000 unidades (18\%) simples que fueron las únicas de toda la muestra. Asimismo, se consumieron 3058000 envases $(70 \%)$ de multivitaminas en comparación con 1293000 envases $(30 \%)$ de vitaminas simples (A11).

Los principios activos más frecuentes en la muestra fueron, en orden descendiente, el mentol (11 especialidades), el ácido acetilsalicílico (8), el alcanfor (6), el ácido ascórbico (6), el salicilato de metilo (6) y la cafeína (5).

\section{Grupos terapéuticos (clasificación ATC)}

En la figura 1 se presenta la distribución de las especialidades vendidas por grupos terapéuticos. La muestra se compuso en su mayor parte de fármacos que actúan sobre el aparato digestivo y el metabolismo (A), la piel (D) o el aparato respiratorio (R).

El número de unidades vendidas por subgrupo terapéutico aparece en la figura 2. El subgrupo terapéutico que alcanzó mayores ventas en unidades fue el de las preparaciones para la garganta. La escasez de tónicos llama la atención: el Biotônico Fontoura ${ }^{\circledR}$ fue el único que se vendió en este subgrupo. Los demás subgrupos con abundantes ventas presentaron un promedio de seis especialidades.

\footnotetext{
3 Los códigos entre paréntesis son los de la clasificación ATC.
}

FIGURA 1. Porcentaje de las ventas de medicamentos en cada grupo terapéutico, de acuerdo con la clasificación ATC

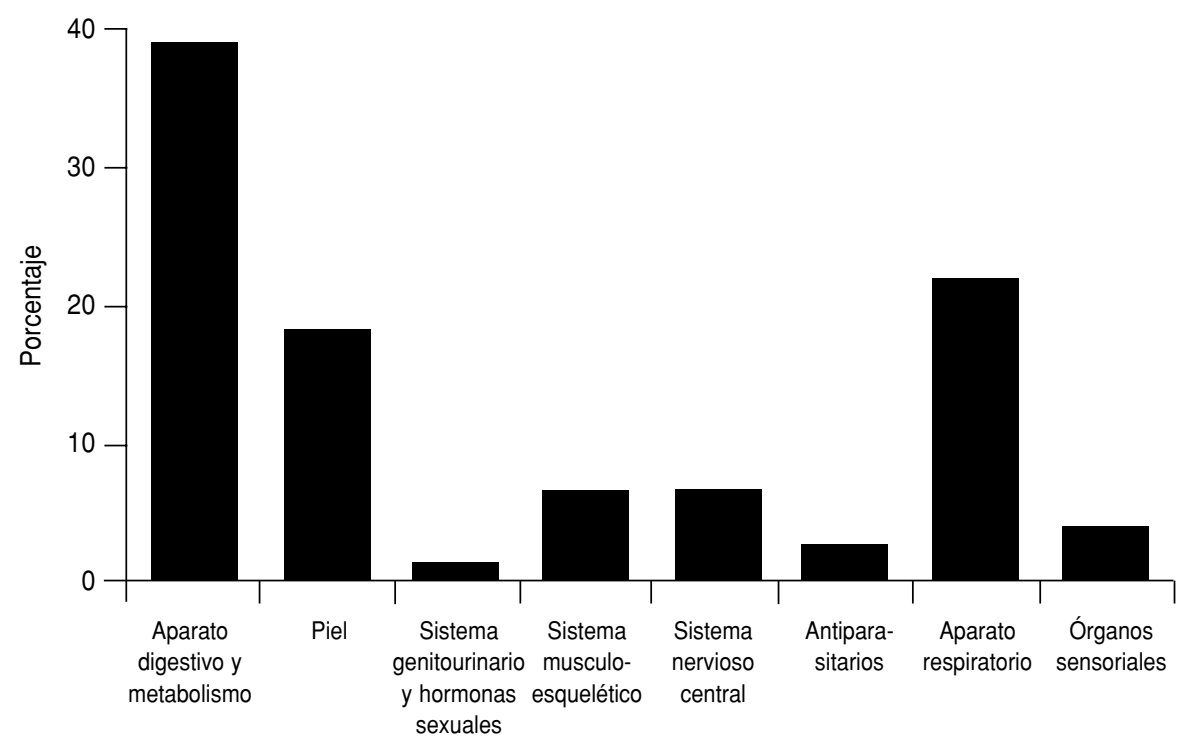

FIGURA 2. Porcentaje de las ventas de medicamentos correspondientes a cada subgrupo terapéutico

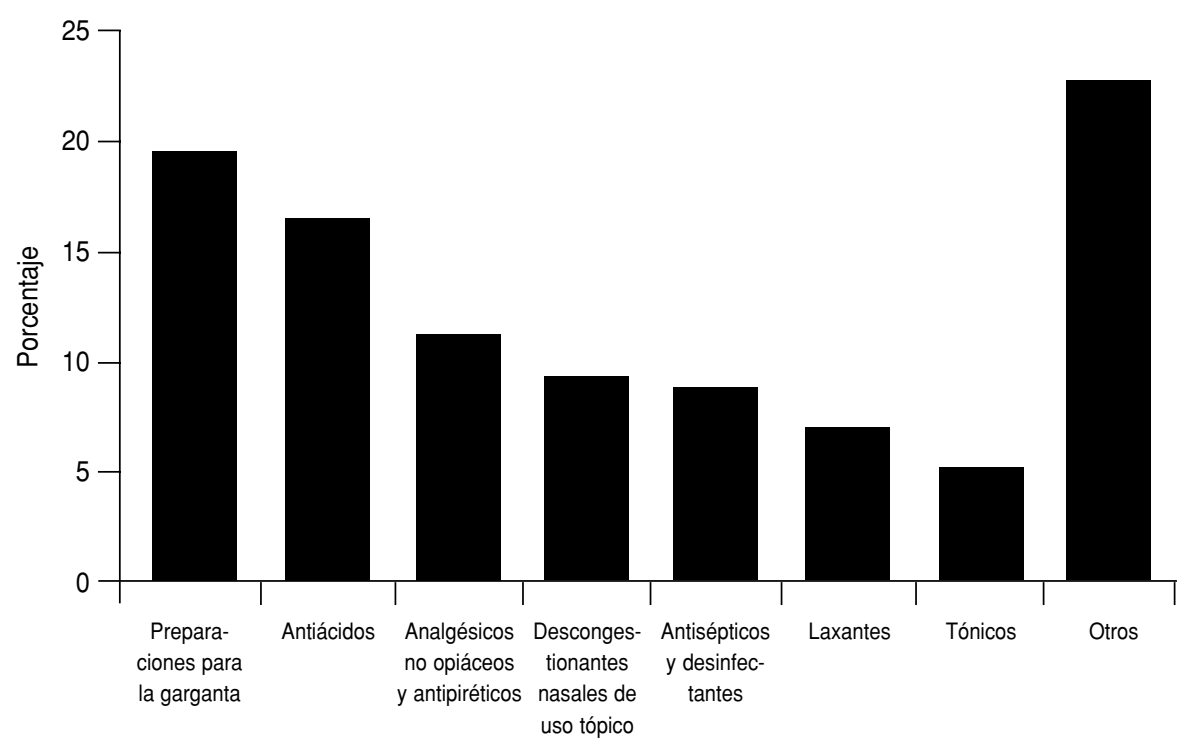

En la figura 3 se presentan los distintos subgrupos terapéuticos que generaron las mayores ganancias en la venta de medicamentos. Los analgésicos no opiáceos y los antipiréticos representaron el mayor valor en ventas.

\section{Valor intrínseco}

En el cuadro 1 se presenta el valor intrínseco de los medicamentos de venta libre en el Brasil, por grupos terapéuticos. Se destaca que $62 \%(48 / 77)$ 
FIGURA 3. Subgrupos terapéuticos que generaron las mayores ganancias en ventas

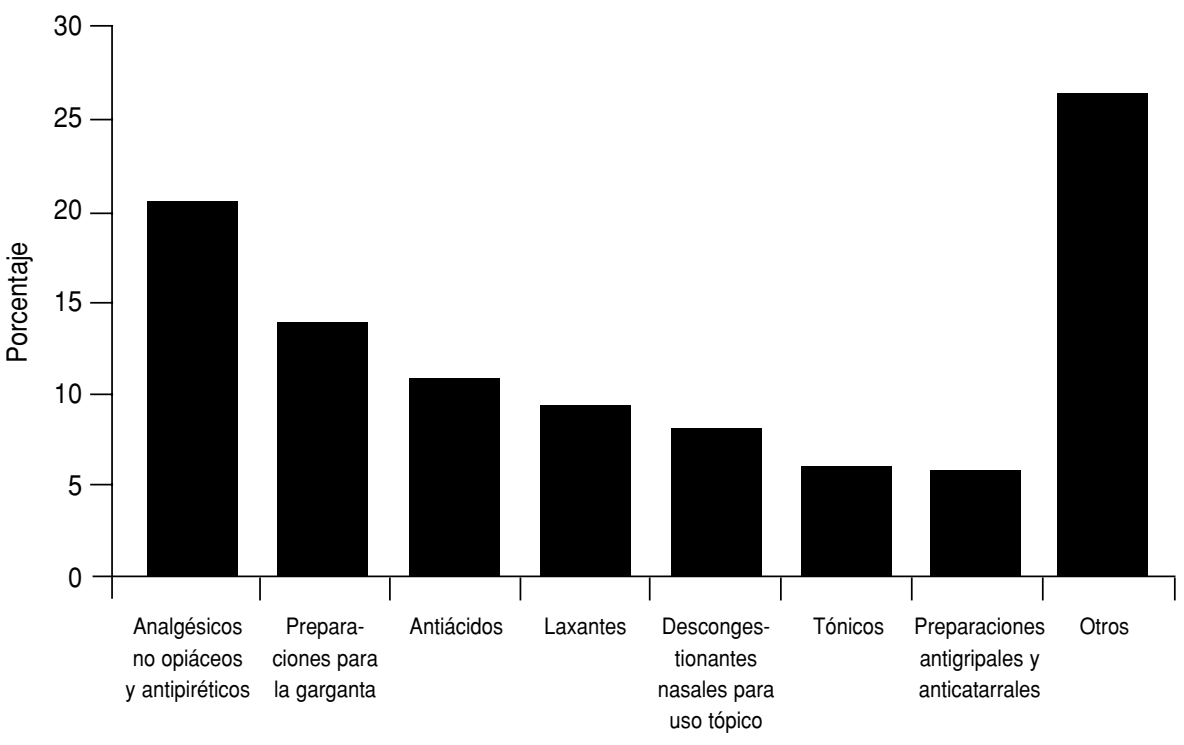

son de valor dudoso o carecen de valor, $19 \%(15 / 77)$ son de valor relativo y $9 \%(7 / 77)$ son inaceptables. Según los criterios utilizados, solamente siete especialidades $(9 \%)$ tienen un alto valor intrínseco y $70(91 \%)$ son de calidad baja o discutible. El gasto en productos de "mala calidad" (aquellos cuyo valor es relativo o dudoso y los que carecen de valor o son inaceptables) correspondió a US\$146541 000, es decir, a $86,9 \%$ del ingreso total generado por todas las ventas.

En el cuadro 1 también se aprecia el valor intrínseco de las especialidades por grupos terapéuticos. Seis de las siete especialidades de alto valor pertenecen al grupo de medicamentos para el aparato digestivo. Este grupo terapéutico también presenta el mayor número de especialidades de valor dudoso o sin valor $(39,6 \%)$ y de fármacos inaceptables $(42,8 \%)$. Destaca el poco valor intrínseco de todas las especialidades para la piel (36) y para el aparato respiratorio (37).

$\mathrm{Al}$ estudiar el valor intrínseco de las especialidades por subgrupos terapéuticos (cuadro 2) se encontraron más de cuatro productos de poco valor entre los preparados vitamínicos (A11), antisépticos desinfectantes (D08) y otros productos dermatológicos (D11), analgésicos tópicos para los dolores musculares y articulares (M02), analgésicos (N02) antigripales y antitusígenos (R05). Solamente se observaron especialidades de alto valor entre los antiácidos, analgésicos no narcóticos y especialmente los laxantes (A06). Sin embargo, los laxantes de alto valor (1 870000 unidades) se vendieron mucho menos que los de poco valor (5 414000 unidades).

\section{Presencia en las listas de medicamentos esenciales del Brasil y de la OMS}

Del total de los 77 medicamentos, solo 10 figuran en la lista de la RENAME y cuatro en la de medicamentos esenciales de la OMS. De los 10 primeros, cuatro se consideran de valor dudoso o sin valor; y de esos cuatro, dos son antisépticos y desinfectantes (D08) cuyo principio activo es el tiomersal, el tercero es un laxante (A06) a base de aceite mineral, y el cuarto es un antidiarreico (A07) a base de tintura de opio.

\section{DISCUSIÓN}

Los resultados de este estudio confirman la hipótesis de que los medicamentos de venta libre en el Brasil son de mala calidad terapéutica y de que el consumo de muchos de ellos redunda

CUADRO 1. Valor intrínseco de los medicamentos de venta libre en el Brasil, por grupos terapéuticos

\begin{tabular}{|c|c|c|c|c|c|c|}
\hline \multirow[b]{2}{*}{ Grupo terapéutico } & \multicolumn{6}{|c|}{ Valor intrínseco } \\
\hline & $\begin{array}{c}\text { Valor relativo } \\
\text { (No.) }\end{array}$ & $\begin{array}{c}\text { Valor dudoso } \\
\text { o ausente } \\
\text { (No.) }\end{array}$ & $\begin{array}{c}\text { Fármaco } \\
\text { inaceptable } \\
\text { (No.) }\end{array}$ & $\begin{array}{l}\text { Total con } \\
\text { poco valor } \\
\text { (No.) }\end{array}$ & $\begin{array}{l}\text { Alto valor } \\
\text { (No.) }\end{array}$ & $\begin{array}{c}\text { Total } \\
\text { general } \\
\text { (No.) }\end{array}$ \\
\hline Aparato digestivo & 2 & 19 & 3 & 24 & 6 & 30 \\
\hline Dermatología & 2 & 11 & 1 & 14 & 0 & 14 \\
\hline Aparato genitourinario & 0 & 0 & 1 & 1 & 0 & 1 \\
\hline Antiparasitarios & 1 & 0 & 1 & 2 & 0 & 2 \\
\hline Aparato respiratorio & 2 & 14 & 1 & 17 & 0 & 17 \\
\hline Órganos sensoriales & 0 & 3 & 0 & 3 & 0 & 3 \\
\hline Total & 15 & 48 & 7 & 70 & 7 & 77 \\
\hline
\end{tabular}


CUADRO 2. Valor intrínseco de los medicamentos por subgrupos terapéuticos

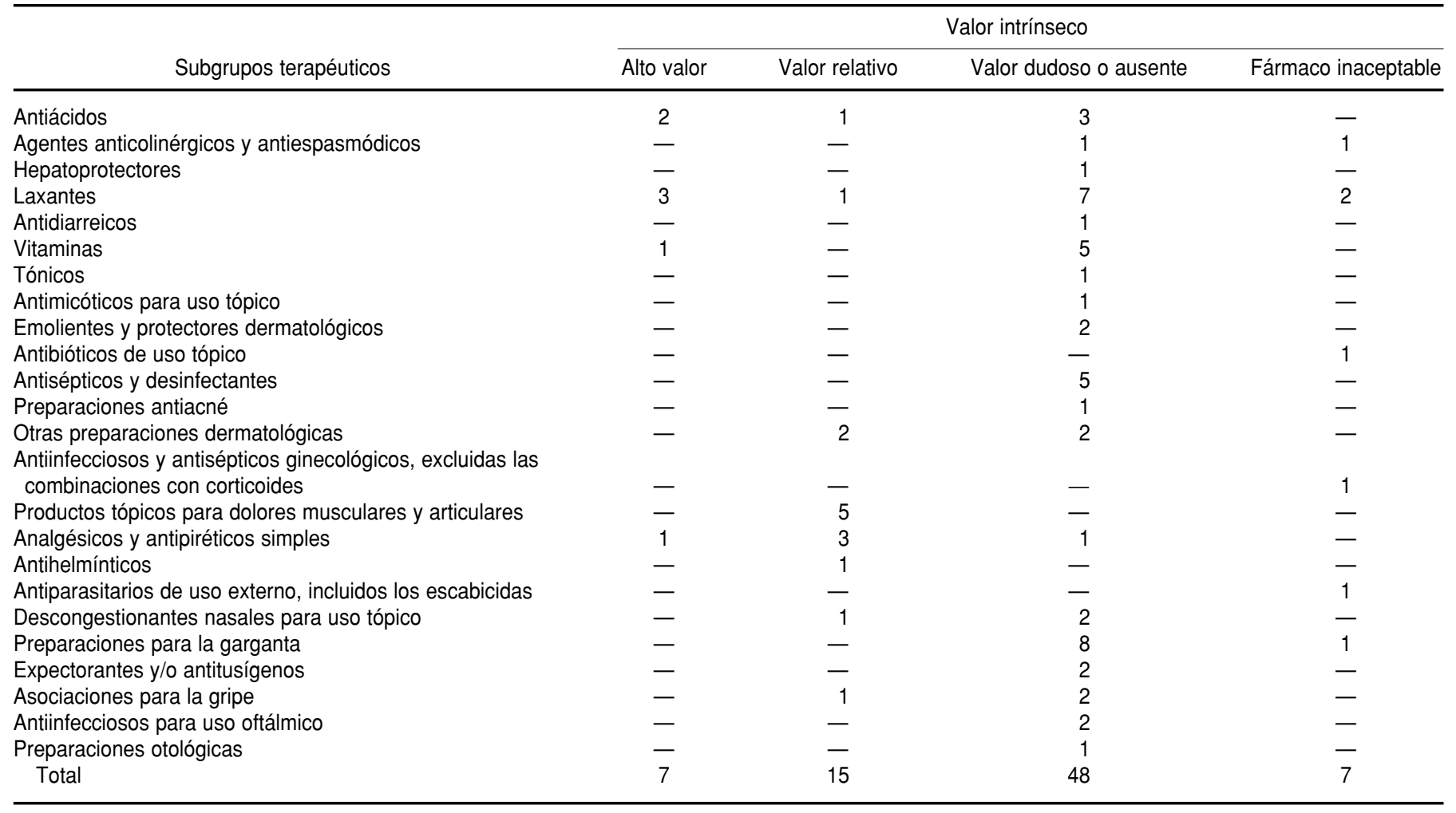

en un gasto innecesario para la población. A estos resultados se suma el hecho de que muchos de los productos examinados son consumidos por decisión exclusiva del paciente y sin ningún conocimiento $u$ orientación sobre su uso y propiedades $(3,38)$.

Respecto a la composición de los productos analizados, se comprobó que las combinaciones a dosis fijas se consumen con mucha más frecuencia que los fármacos simples. Las primeras solo son aceptables cuando aventajan a las segundas en eficacia terapéutica, inocuidad o acatamiento por parte del paciente. Ciertos subgrupos terapéuticos, como los analgésicos, podrían estar representados por medicamentos de alto valor si no fuesen combinados. Por otro lado, algunas asociaciones son irracionales, como la de metoclopramida con antiácidos, o la de fármacos antigripales con analgésicos -en algunas especialidades, a dosis infraterapéuticas-, cafeína, ácido ascórbico, antihistamínicos, simpatomiméticos y antiácidos. El precio de las combinaciones es otro factor que debe tenerse en cuenta. Por ejemplo, las combinaciones de analgésicos cuestan, en promedio, el doble de lo que cuestan los fármacos simples. Si en lugar de Aspirina $C^{\circledR}$ (ácido acetilsalicílico, 400 mg + ácido ascórbico, 240 mg) se consumiera Aspirina ${ }^{\circledR}$ (ácido acetilsalicílico, $500 \mathrm{mg}$ ), se ahorrarían aproximadamente US\$213639 (precio de dos comprimidos de Aspirina ${ }^{\circledR}$ efervescente para adultos: US $\$ 0,46$; de dos comprimidos de Aspirina $C^{\circledR}$ efervescente: U\$ 0,67).

Aunque la gran abundancia de medicamentos de venta libre de poco valor terapéutico hace que la oferta sea inadecuada, este hecho por sí solo no explica su uso irracional. Aunque en este estudio no se exploraron los motivos que llevan al consumo de los distintos medicamentos, es factible, por ejemplo, que algunas formulaciones vitamínicas de alto valor intrínseco hayan sido utilizadas en situaciones en que no estaban indicadas o como sustitutas de una alimentación ade- cuada $\mathrm{y}$, por lo tanto, de una manera irracional.

Algunos de los medicamentos no deberían estar al alcance de personas que desconocen las complicaciones asociadas con su utilización. El país carece de un sistema para la notificación de reacciones medicamentosas adversas, de modo que no hay datos que permitan evaluar la magnitud del problema. Sin embargo, el hecho bien documentado de que los medicamentos povocan la mayor parte de las intoxicaciones registradas en el país (25\%) (39) apunta a la ignorancia en torno a su uso. La metoclopramida es una sustancia que no debería venderse libremente, ya que tiene efectos secundarios nocivos, como reacciones extrapiramidales y otros efectos menos graves, como somnolencia, estreñimiento, diarrea, edema de la mucosa oral y erupciones cutáneas $(20,22)$. Su venta sin receta puede ser perjudicial y su combinación con antiácidos redunda en su uso excesivo. La mayoría de los medicamentos, sin embargo, no presentan 
reacciones adversas graves ni frecuentes y su peor consecuencia es quizás la inversión de los escasos recursos económicos en productos ineficaces o innecesarios. Ello no solamente conlleva un peligro para la salud, sino que también impide que se destinen los recursos económicos, en lo individual y en lo social, a otras medidas más necesarias o al menos más eficaces.

Los resultados de este estudio son perturbadores, pues demuestran que en el Brasil, país donde gran parte de la población es de bajos ingresos, se invierte una enorme cantidad de recursos en la compra de medicamentos de escaso valor terapéutico. Además, muy pocas de las especialidades de la muestra estudiada se consideran esenciales. Es necesario estimular la prescripción, producción, comercialización y utilización de los medicamentos que sí son esenciales y reexaminar la RENAME, que actualmente se encuentra al margen de las políticas de salud del país.

En las últimas décadas, las medidas gubernamentales en torno al registro y a la vigilancia de los medicamentos en el Brasil han sido ineficaces. Ninguna ha logrado mejorar la oferta de medicamentos o adecuar la publicidad de los medicamentos de venta libre a la legislación existente. Desde los años sesenta tres comisiones parlamentarias han investigado el asunto de los medicamentos y han encontrado diversas irregularidades $(37,40,41)$. Se ha diag- nosticado el problema, pero no se ha avanzado hacia su resolución. Más recientemente se han adoptado algunas medidas correctoras: se exige poner el nombre genérico en la receta y que el profesional farmacéutico esté presente en la farmacia durante todas las horas en que se atiende al público (41).

En febrero de 1993, la Secretaría Nacional de Vigilancia Sanitaria (SNVS) trató de mejorar la oferta de medicamentos promulgando una interdicción cautelar por 90 días de 34 medicamentos (portaria SNVS no. 10 DOU 11/02/ 93 y no. 11 DOU 13/02/93) sin aparente utilidad (41). La gran mayoría de estos medicamentos eran de venta libre. Muchos laboratorios farmacéuticos recurrieron a la justicia y consiguieron que sus productos se eliminaran de esta lista y se vendieran de nuevo (42). Recientemente (agosto de 1997), las portarias no. 165/97 y no. 172/97, ambas de la SNVS, han prohibido la producción, comercialización y venta de antibióticos combinados que no tienen una justificación científica. En general, las leyes nacionales y las medidas reguladoras son adecuadas, pero no se cumplen o no perduran.

Independientemente del éxito que alcancen las medidas destinadas a mejorar la oferta de medicamentos, una estrategia imprescindible para mejorar la utilización de estos en el largo plazo es modificar la percepción que tiene la población en general con respecto a la necesidad de usar el medicamento para tratar sus dolencias. Esto ya ha ocurrido en relación con los laxantes $\mathrm{y}$ antidiarreicos, que actualmente son utilizados con mucha más cautela. Si se cambia la actitud de la población frente a los medicamentos, el mercado tendrá que adaptarse. Este cambio se puede lograr con la difusión de información y con el apoyo de los profesionales de la salud, principalmente de los médicos y farmacéuticos que recetan o venden estos productos.

Las organizaciones no gubernamentales también aportan un apoyo fundamental y representan los intereses de la sociedad en general. Educar y proteger a la población se hace todavía más necesario frente a la fuerza de la publicidad utilizada por los fabricantes para generar las ventas a base de fomentar la automedicación sin la debida información y con productos inadecuados (43). También depende de esos profesionales, principalmente los afiliados con instituciones académicas y públicas, realizar estudios sobre los diversos aspectos de la utilización de los medicamentos. Se necesita mucha información sobre el tema para poder orientar futuras intervenciones y revisar con ojos críticos el registro de medicamentos del país. Los estudios sobre la utilización de medicamentos sirven para conocer los medicamentos que desea y necesita la población y permiten idear campañas educativas sobre el uso correcto de los mismos y sobre la atención de la salud en general.

\section{REFERENCIAS}

1. Haines A. Health care in Brazil. Br Med J 1993; 306:503-506.

2. Campos GWS. A saúde pública e a defesa da vida. 2a edición. São Paulo: Editora Hucitec; 1994.

3. Greco DB, Leal SS, Filogênio CJB. Medicamentos sin receta. Bol Oficina Sanit Panam 1986;101:45-49.

4. Schenkel EP, et al. Comercialização de medicamentos em bares/lancherias e armazéns/ fruteiras em Porto Alegre. Ciencia Cultura 1990;40:285-288.

5. Schulte-Sasse H. Los cien años de mercado en Latinoamérica. Amsterdam: BUKO-PharmaKampagne; 1993.

6. Coelho HLL. Misoprostol and illegal abortion in Fortaleza, Brazil. Lancet 1993; 341:1261-1263.
7. Costa SH, Vessey M. Misoprostol and illegal abortion in Rio de Janeiro, Brazil. Lancet 1993; 341:1258-1261.

8. Melo JMS. Dicionário Brasileiro de Especialidades Farmacêuticas. 14a edición. São Paulo: Epume; 1992-93.

9. Brasil, Ministério da Saúde. I, Catálogo Brasileiro de Produtos Farmacêuticos. Rio de Janeiro: Ministério da Saúde; 1984.

10. Organización Mundial de la Salud. Guidelines for ATC classification. Oslo: Centro Colaborador de la OMS para la Metodología de las Estadísticas Farmacéuticas; 1990.

11. Laporte JR, Porta M, Capellà D. Drug utilization studies: a tool for determining the effectiveness of drug use. Br J Clin Pharm 1983; 16:301-304.
12. American Medical Association. AMA drug evaluations. 6th edition. Philadelphia: W.B. Saunders; 1986.

13. Anónimo. FDA bans 223 ingredients in nonprescription drug products. Clin Pharmacol 1991;10:92-93.

14. Institut Català de la Salut. La gastroenteritis aguda. Butlletí d'informació terapèutica. 1987; 1(1):1-4.

15. Ahmad SR. USA: Bans on 415 ineffective drug ingredients. Lancet 1992;340:598-599.

16. Institut Català de la Salut. Tractament de la dispèpsia i de la malaltia ulcerosa. Butlletí d'informació terapèutica 1988;2(6):33-38.

17. Institut Català de la Salut. Tractament de la constipació. Butlletí d'informació terapèutica. 1990; 4(5):21-25. 
18. Chetley A. Medicamentos problemas. 2a edición. Amsterdam: Acción Internacional para la Salud 1994.

19. Chetley A. Peddling placebos: an analysis of cough and cold remedies. Amsterdam: Health Action International; 1989.

20. Acadèmia de Ciències Mèdiques de Catalunya i de Balears. Índex Farmacològic 1992. Barcelona: Acadèmia de Ciències Mèdiques de Catalunya i de Balears; 1987.

21. Engle JP, Donnely AJ. Selecting a nonprescription internal analgesic. Amer Pharm 1993; 33:28-34.

22. Gilman AG, Rall TW, Nies AS, Taylor P, eds. As bases farmacológicas da terapêutica. 8a edición. Rio de Janeiro: Guanabara-Koogan; 1990.

23. American Pharmaceutical Association. Handbook of nonprescription drugs. 9th edition. Washington, DC: American Pharmaceutical Association; 1990.

24. Hartog R, Schulte-Sasse H. German and Swiss drug supplies to the Third World. Bielefeld: BUKO Pharma-Kampagne.

25. Kauffman RE, Banner W, Berlin C. Camphor revisited: focus on toxicity. Pediatrics 1994;94: $127-128$.

26. Leung A. Encyclopedia of common natural ingredients. New York: John Wiley \& Sons; 1980.

27. Liebelt E, Michael W, Shannon S. Small doses, big problems: a select review of highly toxic common medications. Pediatr Emerg Care 1993;9:292-297.
28. Physician's desk reference. 45th edition. Oradel: Medical Economics; 1995.

29. Reynolds JEF. Martindale: the extra pharmacopoeia. 30th edition. London: The Pharmaceutical Press; 1993.

30. Schenkel EP, Petrovick PR, Linck VO, Chaves CG, Gosmann G, Costa C, org. Cuidados com os medicamentos. Porto Alegre: Sagra-DC Luzzato; 1991.

31. Schenkel EP, Mengue S. Vitaminas: associações irracionais. Ciencia Cultura 1990; 42:986-989.

32. Smith MBH, Feldman W. Over-the-counter cold medications: A critical review of clinical trials between 1950 and 1991. JAMA 1993;269: 2258-2263.

33. United States Pharmacopeia. USP-DI. 10th edition. Rockville, MD: United States Pharmacopeial Convention, Easton: Mack; 1990.

34. Valladares G. Hepatoprotectores. Med Salud Popular 1992;19:15-18.

35. Brasil, Ministério da Saúde. Memento Terapêutico CEME 89/90. Brasília: Central de Medicamentos; 1989.

36. Organización Mundial de la Salud. Uso de medicamentos esenciales: sexto informe del Comité de Expertos de la OMS. Ginebra: OMS; 1995. (Serie de Informes Técnicos, No. 850).

37. Machado U. 20 anos da indústria da doença. Porto Alegre, Brasil: Civilização Brasileira; 1982.

38. Arrais PS, Coelho HLL, Batista MCDS, Carvalho ML, Righi RE, Arnau JM. Perfil da automedicação no Brasil. Rev Saude Publica 1997;31(1):71-77.

39. Brasil, Ministério da Saúde. Relatório de intoxicações humanas, 1994. Rio de Janeiro: Sistema Nacional de Infomações Toxico-Farmacológicas; 1996.

40. Machado U. A indústria farmacêutica no Brasil. Rio de Janeiro: Departamento de Imprensa Nacional; 1963

41. Sociedade Brasileira de Vigilância de Medicamentos. Medicamentos "inócuos", fajutos, inúteis e essenciais. Boletim SOBRAVIME 1993;9:2.

42. Anónimo. More turmoil in Brazilian pharma sector. SCRIP (World Pharm News);1799:18.

43. Heineck I, Gallina SM, Silva T, Dal Pizzol F, Schenkel EP. Análise da publicidade de medicamentos veiculada em emissoras de rádio do Rio Grande do Sul. Cad Saude Publica 1998; 14(1). En preparación.

Manuscrito recibido el 17 de octubre de 1996 y aceptado para publicación en versión revisada el 19 de noviembre de 1997

ABSTRACT In this study we examined the 100 over-the-counter drugs that were most widely sold in Brazil from June 1992 to June 1993. We excluded 23 food products that were classified as medicinal. The sample, which included 77 drugs, was representative of about $67 \%$ of all sales in monetary value, and $76.8 \%$ of all units sold of the different classes of over-the-counter drugs. The anatomical-therapeutic-chemical (ATC) system was used to classify the various drugs; each class of drugs was also graded according to a scale of its intrinsic value, taking into account effectiveness and risks. Most classes of drugs (91\%) showed "little intrinsic value" (that is, were of questionable or no value, of relative value, or unacceptable), and $70 \%$ were fixed-dose combinations. Only 10 classes of drugs were included in the Ministry of Health's National Drug List, and four appeared in WHO's list of essential drugs. The therapeutic classes to which the drugs belonged were, in descending order of frequency, for the digestive tract, skin, genitourinary system, musculo-skeletal system, central nervous system, parasitic diseases, respiratory tract, and sensory system. The therapeutic subgroup that commanded the highest sales was that of the non-opiate analgesics and antipyretics. Our results confirm the hypothesis that over-the-counter drugs in Brazil are of poor therapeutic quality and that the use of many such drugs is a source of unnecessary expense for the population. Some of them should not be accessible to individuals who lack current knowledge of their side effects, since $25 \%$ of all cases of poisoning in the country are related to self-medication. Modifying the public's perception of the need for taking drugs to relieve their ailments is one approach that may improve their use of medications in the long term. 